\title{
Chlorine Dioxide Sterilization
}

National Cancer Institute

\section{Source}

National Cancer Institute. Chlorine Dioxide Sterilization. NCI Thesaurus. Code C101691.

A sterilization process that uses chlorine dioxide at ambient temperatures to kill microorganisms. This process can be used in large spaces. 